\title{
Orbital Comptonization in accretion disks around black holes
}

\author{
P. Reig ${ }^{1,2}$, N. D. Kylafis ${ }^{1,2}$, and H. C. Spruit ${ }^{3}$ \\ 1 University of Crete, Physics Department, PO Box 2208, 71003 Heraklion, Crete, Greece \\ ${ }^{2}$ Foundation for Research and Technology-Hellas, 71110 Heraklion, Crete, Greece \\ 3 Max-Planck-Institute for Astrophysics, Box 1317, 85741 Garching, Germany
}

Received 4 April 2001 / Accepted 7 June 2001

\begin{abstract}
We have performed Monte Carlo simulations of Compton upscattering of low-energy photons in an accretion disk around a Schwarzschild black hole. The photons gain energy from the rotational motion of the electrons in the disk. The upscattering occurs near the black hole horizon, where the flow velocity of the electrons approaches the speed of light. We show that this type of bulk-flow Comptonization can produce power-law Xray spectra similar to the ones observed in black-hole X-ray transients in the high/soft state, i.e., a soft bump dominating the spectrum below $\sim 10 \mathrm{keV}$ and a power-law tail with photon index in the range $2-3$. In order to reproduce the observed hard to soft flux ratio the disk has to have vertical optical depth above $\sim 3$ at the last stable orbit. We conclude that the power-law component of the high/soft state of black-hole transients may be due to an intrinsically cool disk extending all the way to the hole, without a separate hot plasma component.
\end{abstract}

Key words. accretion, accretion disks - black hole physics - radiation mechanisms: non-thermal methods: statistical - X-rays: stars

\section{Introduction}

The X-ray continuum spectrum of black-hole binaries is characterized by an ultrasoft component and a powerlaw hard tail. The ultrasoft component is interpreted as coming from an optically thick accretion disk. The temperature of the disk is high $(\sim 1 \mathrm{keV})$ near the black hole and decreases outwards. Thus, the spectrum emitted by the disk is a multi-temperature black-body spectrum. The power-law tail is thought to originate from Comptonization of soft photons scattering off very energetic electrons. The amplitude of this component has been seen to correlate with the $1-10 \mathrm{keV}$ flux. During the low (also called hard) state, the ultrasoft component is very weak or absent, whereas in the high (soft) state it dominates the spectrum, especially at energies $\lesssim 10 \mathrm{keV}$.

Two types of Comptonization processes have been put forward to explain the power-law component: thermal and bulk-flow Comptonization. Thermal Comptonization requires the presence of a hot and rarefied cloud of electrons or corona (see, e.g., the review by Poutanen 1998 and references therein), where the soft photons get upscattered due to collisions with Maxwellian distributed 50-100 keV electrons. In the bulk-flow Comptonization scenario (see, e.g., the review by Kylafis \& Reig 1999; Papathanassiou \& Psaltis 2001), photons gain energy from collisions with ra-

Send offprint requests to: P. Reig, e-mail: pablo@physics.uoc.gr dially infalling electrons. Titarchuk et al. (1997) assessed the relative importance of these two processes and concluded that the bulk motion is more efficient in upscattering photons than thermal Comptonization in spherical accretion, provided that the electron temperature in the radial flow is less than a few $\mathrm{keV}$.

Although these Comptonization models have been succesful in describing the spectra of black-hole candidates, there are still a number of unresolved issues. A radial inflow of plasma has been considered for mathematical simplicity, but how this radial inflow is formed is unclear. Since the disk is likely to have a magnetic field generated by the shear flow, it may well have a corona heated by magnetic dissipation (Galeev et al. 1979). Comptonization in this hot corona is one of the currently popular models for the hard spectral components in X-ray binaries, the other being two-temperature flows or "ADAFs". There is little independent evidence for either of these possibilities, however, apart from the interpretation of the X-ray spectrum they offer.

On the other hand, general agreement exists on the presence of the accretion disk itself around a black hole in a mass transfering binary system. In this work we investigate the Comptonization process in a cold accretion disk around a black hole by Monte Carlo simulations. Soft photons produced inside or outside the disk get upscattered by collisions with fast-moving electrons, which follow circular trajectories outside the last stable orbit and ballistic 


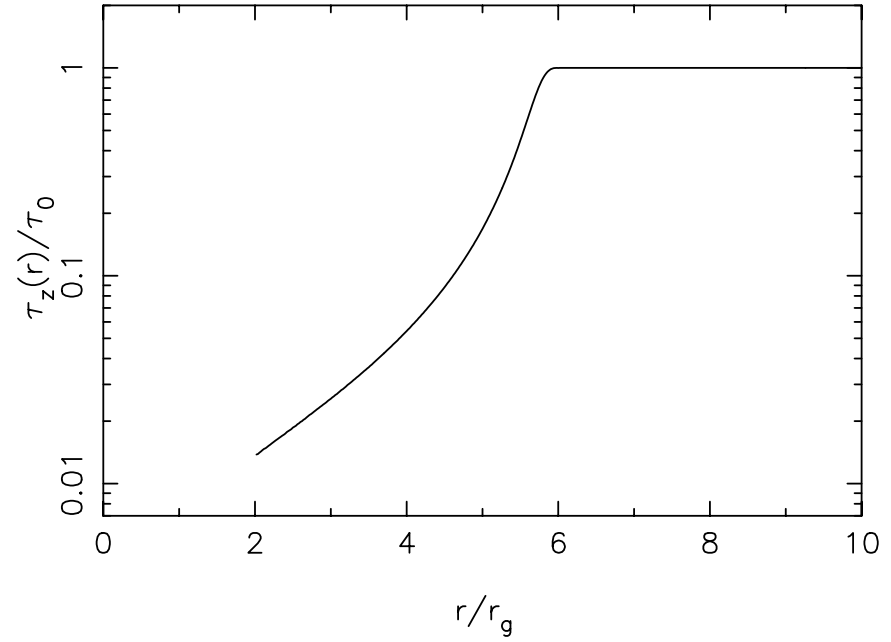

Fig. 1. Variation of the vertical optical depth with radius in the inner disk (normalized to the optical depth $\tau_{0}$ in the outer parts of the disk), for the model used in the scattering calculations.

trajectories inside it. We refer to this process by the name "orbital Comptonization". It has been considered before by Hanawa (1990) who applied it to the boundary layer between a disk and a neutron star surface.

\subsection{Thermal vs. bulk-flow Comptonization}

In a geometrically thin disk, the scattering photons escape easily before they have a chance to pick up energy from the orbital motion. Bulk-flow Comptonization is therefore likely to work better in thicker disks. Such disks are also hotter however, so that thermal Comptonization will also take place.

To compare the relative contributions of the two processes, note that the scattering photons drift from their point of origin by random walk. Before they escape through the disk surface, they therefore sample the orbital velocity field over a radial distance which is of the same order as the disk thickness. The typical velocity difference encountered by a scattering photon is thus of the order $\bar{v}=\Omega H \sim c_{\mathrm{s}}$, where $c_{\mathrm{s}}$ is the sound speed in the disk and $\Omega$ is the angular velocity. In the absence of radiation pressure, $c_{\mathrm{s}} \sim\left(k_{\mathrm{B}} T / m_{\mathrm{p}}\right)^{1 / 2}$. In thermal equilibrium, the thermal velocity of the scattering electrons is $c_{\mathrm{s}}\left(m_{\mathrm{p}} / m_{\mathrm{e}}\right)^{1 / 2} \sim\left(m_{\mathrm{p}} / m_{\mathrm{e}}\right)^{1 / 2} \bar{v} \gg \bar{v}$. It would thus seem that thermal Comptonization wins over bulk-flow Comptonization by the orbital motion. For cool ShakuraSunyaev disks this would indeed be the case. However, the temperatures in such disks, of the order $1 \mathrm{keV}$, are not sufficient for significant Comptonization anyway.

More interesting are the geometrically thick accretion flows that are possible in the inner regions of disks. Here the conditions are different from those in standard thin disks. In the optically thin ADAF type flows, thermal equilibrium between protons and electrons does not hold, and the scattering electrons are much colder than expected from the thickness of the disk. The electron temperatures are near $100 \mathrm{keV}$ so that the electron velocities are around $0.5 c$. This is of the same order as the range of orbital velocities sampled by a photon escaping through a disk of thickness $H / r \sim 1$ near the last stable orbit. In the optically thin type of ADAF, orbital Comptonization can therefore be as important as thermal Comptonization, though it may not dominate.

Another type of radiatively inefficient accretion flow into a black hole takes place when the optical depth of the flow is large, and the disk is puffed up to aspect ratios $H / r \sim 1$ by radiation pressure. The temperature in such radiatively supported disks is relatively low, so that thermal Comptonization is not very effective, but the escaping photons will have sampled a large range of orbital velocities. The conditions in optically thick radiation supported flows such as may be present at high accretion rates onto black holes are thus especially relevant for orbital Comptonization.

\section{The model}

\subsection{Densities and velocity field}

In an accretion disk around a non-rotating black hole of mass $M$ matter follows nearly circular orbits only up to the last stable orbit at a distance of $r_{\text {last }}=6 r_{\mathrm{g}}$, where $r_{\mathrm{g}}=G M / c^{2}$ is the gravitational radius. At the black hole horizon $r_{\mathrm{h}}=2 r_{\mathrm{g}}$, the speed of the particles is $c$.

The details of bulk Comptonization depend on the velocity field of the accretion flow, as well as its optical depth and geometrical thickness as a function of the distance from the hole. For the high accretion rates we have in mind, the flow has a finite thinkness of the order $r_{\mathrm{g}}$, depending on the details of disk viscosity and radiative transfer. Instead of a more detailed quantitative model, we approximate the velocity field by purely circular motion outside the last stable orbit, and a ballistic spiral-in inside $r=r_{\text {last }}$, as follows.

For $r \geq r_{\text {last }}$, matter in the disk is taken to be in circular motion. In Schwarzschild coordinates, the orbital frequency as seen by a distant inertial observer is then $\Omega=\left(G M / r^{3}\right)^{1 / 2}$ (Misner et al. 1973 (hereafter MTW)), the same as in Newtonian gravity. At $r=r_{\text {last }}$ and in geometric units, the energy of the particles per unit mass is $E=\sqrt{8 / 9}$ and their angular momentum per unit mass $L$ divided by the black-hole mass $M$ is $L / M=\sqrt{12}$ (MTW, p. 662).

To find the velocity of the gas as a function of $r$ as it falls ballistically into the black hole, we use the expressions (Shapiro \& Teukolsky 1983)

$v_{\phi}(r)=\frac{L}{r E} \sqrt{1-\frac{2 M}{r}}$
$v_{\mathrm{r}}(r)=\frac{1}{E} \sqrt{E^{2}-\left(1-\frac{2 M}{r}\right)\left(1+\frac{L^{2}}{r^{2}}\right)}$.

We assume that just inside $r_{\text {last }}, E=\sqrt{8 / 9}$ and $L / M=$ $\sqrt{12}-\epsilon$, where $\epsilon \ll \sqrt{12}$ is a measure of the radial drift 


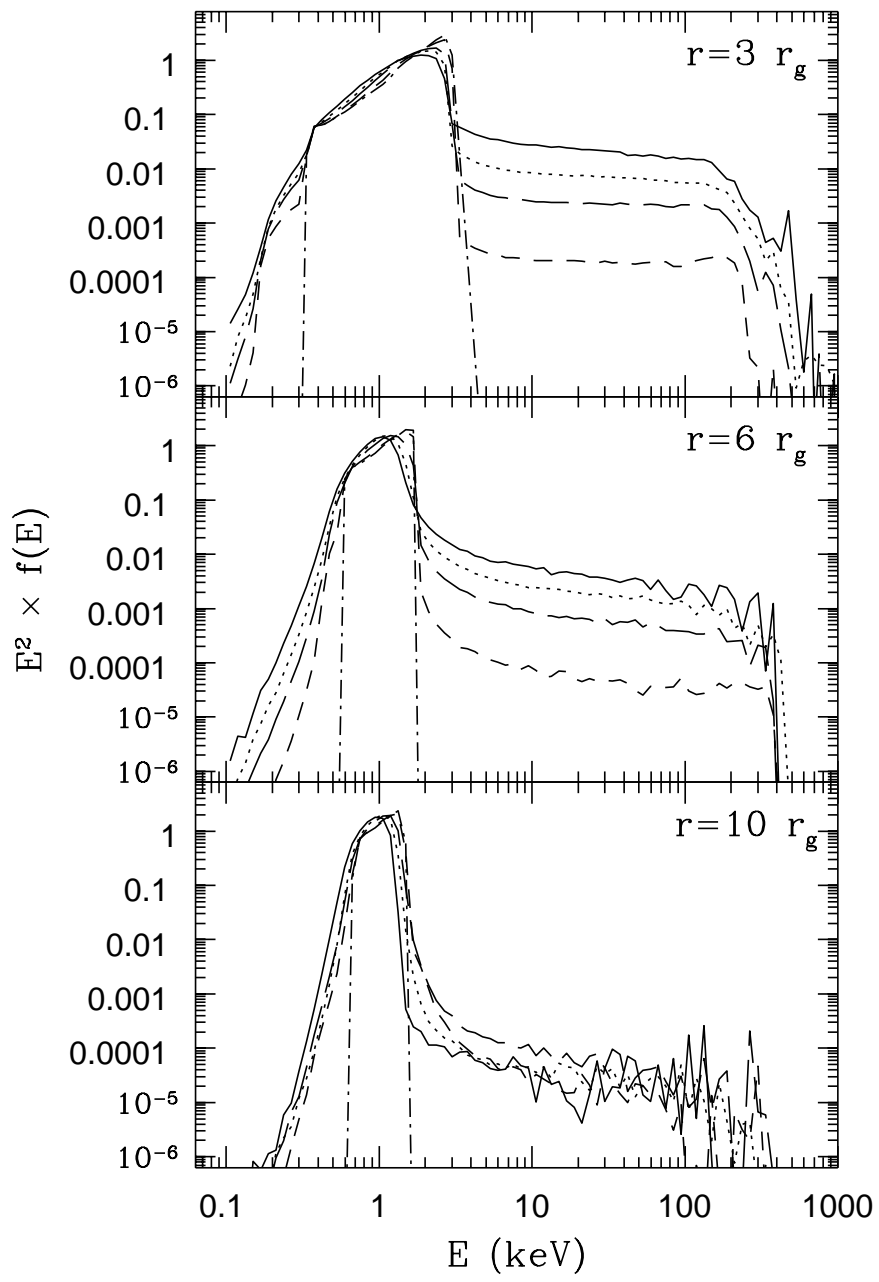

Fig. 2. Emergent spectral energy distributions as functions of position $r / r_{\mathrm{g}}$ of the source of soft photons (monochromatic at $1 \mathrm{keV}$, in the midplane of the disk), and $\tau_{0}$, a measure of the vertical optical thickness (see text and Fig. 1). $\tau_{0}=0$ (dotdashed), 0.1 (short-dashed), 1 (long-dashed), 3 (dotted), and 10 (solid). The three panels are for $r=3 r_{\mathrm{g}}$ (top), $r=6 r_{\mathrm{g}}$ (middle) and $r=10 r_{\mathrm{g}}$ (bottom).

speed in the accretion disk just outside the last stable orbit. The exact value of $\epsilon$ has no effect on the calculations.

To compute the density and the vertical optical thickness of the flow, we assume a constant thickness $H=2 r_{\mathrm{g}}$ (as seen by a distant observer). From the continuity equation and the assumed velocity field, the vertical optical depth can then be computed from the radial velocity (Eq. (2)). Outside $r_{\text {last }}$, where we have assumed circular orbits, we take a constant density. The resulting vertical optical depth of the disk as a function of distance, $\tau_{z}(r)$, is shown in Fig. 1.

Bulk-flow Comptonization that produces hard X-rays is effective only near the black-hole horizon, where the speed of the electrons is high. Thus, only those photons that find themselves in the inner part of the disk, and manage to escape eventually, will have their energy significantly increased. It is these photons that produce the high-energy power-law spectra.

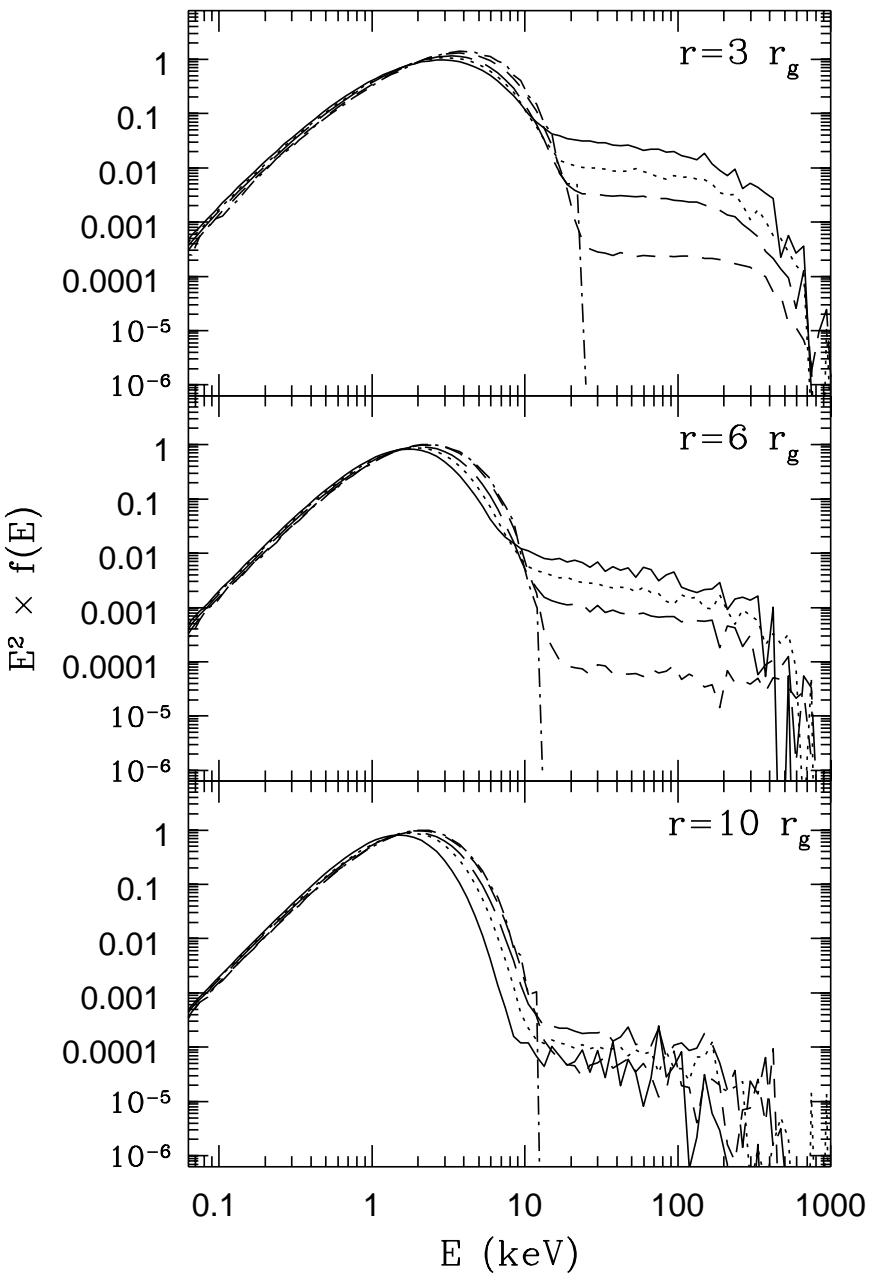

Fig. 3. Same as in Fig. 2, but with a blackbody source (dotdashed line) of soft photons of temperature $k T=0.5 \mathrm{keV}$ instead of a monochromatic source.

\section{Radiative transfer}

Photons are generated throughout the flow, diffuse over some distance and leave it at some other point. The radiation leaving the disk at any point of its surface is thus an integral over the contributions from all source positions. In order to highlight the importance of different source positions (in particular, of their distance to the hole), we show here results for fixed source positions. The net spectrum can be obtained from this by integration, if needed. The emission of photons at the source is assumed to be isotropic in a comoving frame, as it would be for photons generated by bremsstrahlung, for example.

As a photon travels in the disk, it experiences Compton scatterings with the orbiting electrons. If the optical depth is small $(\lesssim 1)$, the majority of the input photons escape unscattered. Those that are directed towards the black hole get absorbed. Those that scatter a few times have on average their energy significantly increased. If the optical depth is moderate, then those photons that are not absorbed by the black hole random-walk through the medium prior to escape, and gain energy from the bulk motion of the electrons. 


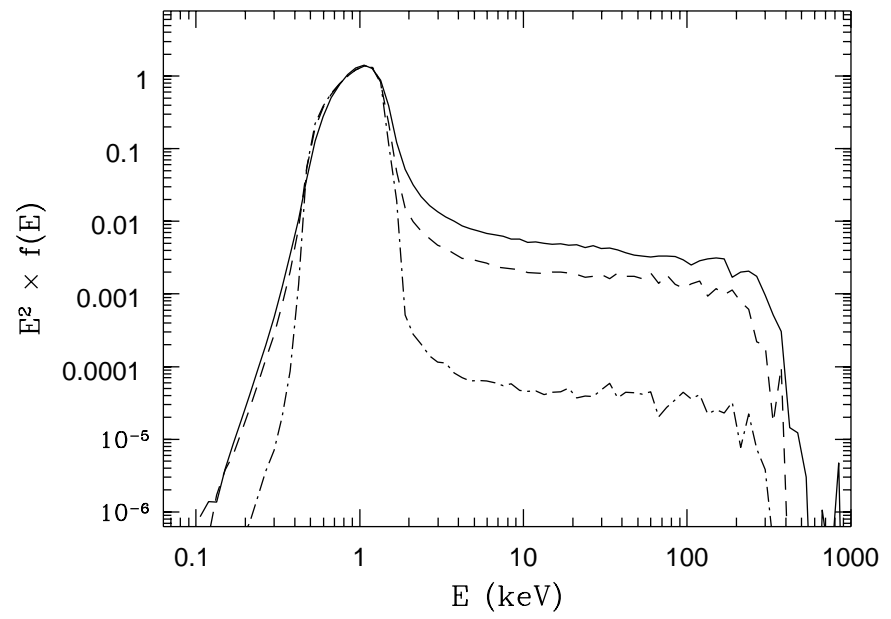

Fig. 4. Dependence of the spectrum on disk thickness, $H=0.3 r_{\mathrm{g}}$ (dot-dashed line), $1 r_{\mathrm{g}}$ (dashed line) and $5 r_{\mathrm{g}}$ (solid line). The input source of photons is monochromatic at $1 \mathrm{keV}$ and is located at $6 r_{\mathrm{g}}$. The optical depth was taken to be 3 .

We make some simplifications in dealing with the general relativistic aspects of the problem. The effect of curvature on the path of the photon between scatterings is neglected, that is, we approximate it as a straight-line as seen by a distant observer. Since the distances traveled between scatterings are small, this is not a bad approximation. For a quantification of the small error made with the use of straight-line photon trajectories the reader is referred to Papathanassiou \& Psaltis (2001). The effect of curvature on the geometrical distances is also neglected since the actual physical distance between scatterings is not important for the scattering process, only the physical conditions at the scattering centers. Finally, we leave out the self-illumination of the disk surface by other parts of the disk (through gravitational light bending, for example).

We do take into account the important effect of the changing gravitational redshift along the photon trajectory. The energy $E\left(r_{1}\right)$ of a photon, as measured by a locally inertial observer at radius $r_{1}$, is related to the energy of the same photon $E\left(r_{2}\right)$ at radius $r_{2}$, as measured by a locally inertial observer through the difference in gravitational redshift (MTW p659)

$E\left(r_{1}\right) \sqrt{1-2 r_{\mathrm{g}} / r_{1}}=E\left(r_{2}\right) \sqrt{1-2 r_{\mathrm{g}} / r_{2}}$.

\subsection{Monte Carlo implementation}

The basic principle of the Monte Carlo technique, as it is applied to the Comptonization process (Cashwell \& Everett 1959; Pozdnyakov et al. 1983), consists of following the life histories of a large number of photons from the moment each photon is emitted from a given source until it leaves the scattering medium.

The photon is characterized by 4 parameters (position, direction, energy and weight) which are updated at each scattering, and determine the spectrum of the radiation

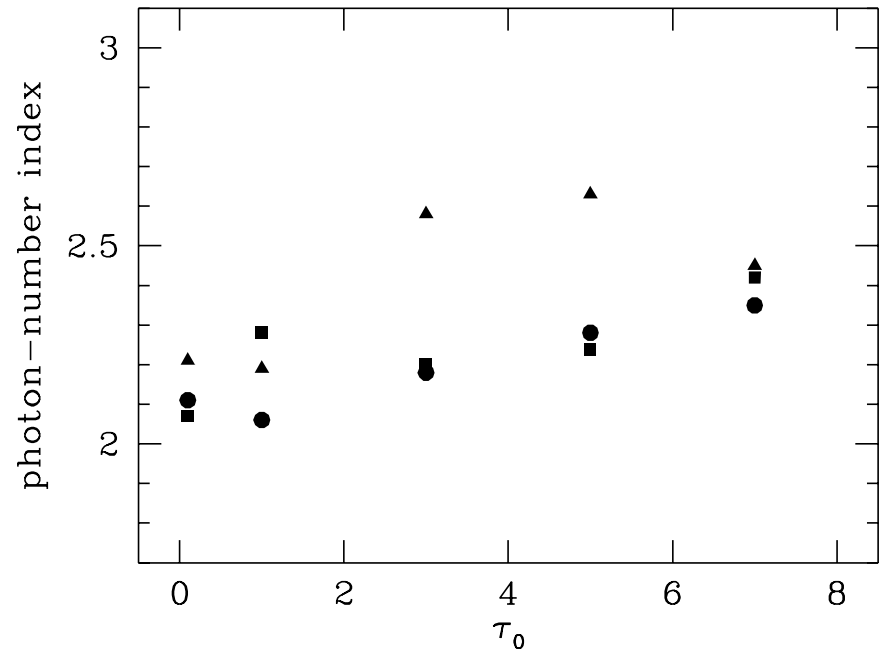

Fig. 5. Photon-number spectral index in the range $20-100 \mathrm{keV}$ as a function of vertical optical depth $\tau_{0}=\tau_{z}\left(r_{\text {last }}\right)$ for the spectra shown in Fig. 2: $r=3 r_{\mathrm{g}}$ (circles), $r=6 r_{\mathrm{g}}$ (squares) and $r=10 r_{\mathrm{g}}$ (triangles).

emerging from the scattering medium. The procedure can be divided into the following steps:

1. The disk is divided into concentric cylindrical zones. Each zone is characterized by a mean velocity and a mean density. The number of zones is chosen so as to have a fine zoning of electron velocities in the inner parts of the disk;

2. We calculate the optical depth to electron scattering $\tau(\boldsymbol{r}, \boldsymbol{n}, E)$ from the scattering position $\boldsymbol{r}$ to the boundary of the disk along the direction of the photon $\boldsymbol{n}$. Then, a portion $W \mathrm{e}^{-\tau}$ of the photon's weight escapes and it is recorded, while the remaining weight $W^{\prime}=W\left(1-\mathrm{e}^{-\tau}\right)$ is scattered between $\boldsymbol{r}$ and the boundary;

3. We determine the new scattering position, the energy of the photon after scattering and its new direction.

The process continues with the next scattering until the photon's weight falls below a certain value $\left(10^{-8}\right.$ in our runs), and it is repeated for $N$ photons, where $N$ is a large number (typically $10^{7}$ ).

\section{Results and discussion}

We consider a point source of soft photons in the midplane of the accretion disk. In the local rest frame of the flow, the source emits monochromatic photons of energy $E_{0}=$ $1 \mathrm{keV}$ isotropically. In the frame of a local inertial observer the distribution of emitted photons is not isotropic but forward peaked (in the direction of the flow) and the initial energy of the photons is Doppler shifted and depends on their initial direction.

The resulting spectral energy distributions $E^{2} f(E)$, where $f(E)$ is the photon-number spectrum, are shown in Figs. 2-4. Figure 2 shows results for three distances of the soft photon source from the hole, $r / r_{\mathrm{g}}=3,6$ and 10, 


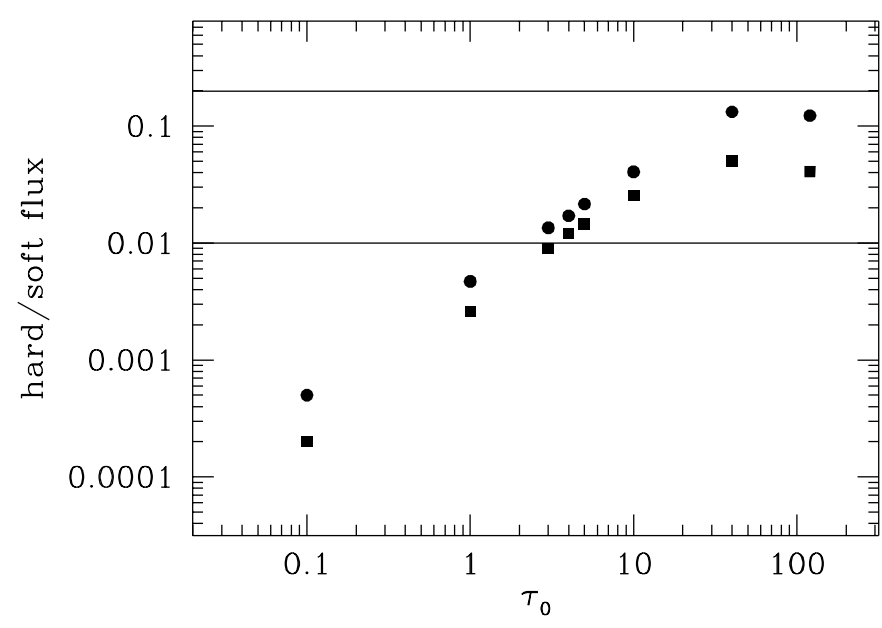

Fig. 6. Hard to soft flux ratio as a function of the vertical optical depth $\tau_{0}=\tau_{z}\left(r_{\text {last }}\right)$ and source position: $r=3 r_{\mathrm{g}}$ (circles), $r=6 r_{\mathrm{g}}$ (squares). The hard and soft bands are $20-200 \mathrm{keV}$ and $1-5 \mathrm{keV}$, respectively. The solid lines enclose the region compatible with observations.

and for five values of the vertical optical depth at the last stable orbit. All five spectra in each panel are normalized so that the integral of $f(E)$ from zero to infinity is equal to 1 .

The emergent spectra in Fig. 2 consist of a peak, whose width increases as the source of photons approaches the black hole, and a high-energy tail that is approximately a power law with a cut off at $100-300 \mathrm{keV}$. The soft peak is formed by those photons that escape unscattered or have had a small energy change before escape. The tail is prominent and extends to high energies even if the optical depth is low. It is formed by those few photons that picked up a lot of energy from the electrons, mainly through headon collisions.

Since model fits to the observed X-ray spectra in blackhole candidates give blackbody temperatures for the accretion disk in the range $\sim 0.3-1 \mathrm{keV}$ (Tanaka \& Lewin 1995), we have also considered a source of soft photons in the disk with blackbody emission of temperature $k T=0.5 \mathrm{keV}$. The pointlike source is again placed at the midplane of the disk. The emergent spectra are shown in Fig. 3. The panels and lines have the same meaning as in Fig. 2.

The amplitude of the high-energy tail and the cutoff photon energy decrease with distance of the photon source from the hole. This is expected since the orbital velocities encountered by the photons decrease with distance. The photon-number spectral index is, however, less dependent on the source position. Within statistical errors, it is also the same for a monochromatic and a black body input spectrum.

Since the scattering photons sample a velocity range that depends on the disk thickness (cf. the discussion in Sect. 1.1), the spectra depend not only on the optical depth, but also on the actual geometric thickness of the disk. This is illustrated in Fig. 4. It shows that the high energy tail is strongly suppressed when the aspect ratio $H / r$ drops substantially below unity.

These spectra are reminiscent of the observed spectra in black-hole candidates in the high/soft state. The resemblance is strengthened when the photon-number spectral index and the ratio of the hard to the soft flux are compared with observations.

The photon-number spectral index in the tail is, within statistical errors, independent of the position of the source of soft photons. It exhibits a weak dependence on the vertical optical depth $\tau_{0}$ at $r=r_{\text {last }}$; the thicker the medium the softer the spectrum (Fig. 5). The index in Fig. 5 was computed from the spectra of Fig. 2, in the energy range between 20 and $100 \mathrm{keV}$. For low and intermediate optical depths, the photon-number index is $\sim 2-2.5$, which is similar to those observed in the high/soft state of black-hole candidates (Tanaka \& Shibazaki 1996).

In addition to the slope of the high-energy tail, we can also compare its strength with the observations. We do this in terms of a hard-to soft flux ratio. Observations of soft state spectra (e.g. Miyamoto et al. 1993; Cui et al. 1997), generally give values of $0.01-0.2$ for the ratio of power law tail to black body fluxes. In Fig. 6 we have plotted the $20-200 \mathrm{keV}$ to $1-5 \mathrm{keV}$ flux ratio of our models as a function of the vertical optical depth $\tau_{0}$. The vertical optical depth presumably correlates with the mass accretion rate as in the case of Comptonization in a free-falling spherical flow (Laurent \& Titarchuk 1999). In our model, however, $\tau_{0}$ is an input parameter that can be changed arbitrarily.

In a realistic accretion disk, it is expected that the majority of the photons in the ultrasoft component come from the region of the disk near $6 r_{\mathrm{g}}$. Close to the black-hole horizon the vertical optical depth of the disk is relatively small and at $r \gg 6 r_{\mathrm{g}}$ the temperature and emissivity of the disk are small. Thus, the squares in Fig. 6 are closer to reality than the circles. In other words, the vertical optical depth of the disk at the last stable orbit must be greater than $\sim 3$ in order for our proposed mechanism to produce spectra similar to the ones observed.

The energy of the photons escaping to infinity is the result of the competition of two processes: the gain of energy due to Compton scattering, which increases towards the horizon, and the loss of energy due to gravitational redshift, which also increases. It can be shown that for a thin disk the escaping photon energy cannot exceed $m_{\mathrm{e}} c^{2}$. Some of the spectra in Figs. 2 and 3 show a cutoff at energies a bit above this limit. This can be traced back to a simplification made in our assumed velocity field. The rotational velocity was taken the same at all $z$ (for the same polar coordinate $\rho$ ). On the other hand, in the gravitational redshift the radial coordinate enters spherically, i.e. $r=\sqrt{\rho^{2}+z^{2}}$, which is larger than $\rho$. In reality, the rotation speed will decrease in step with the redshift.

Some black hole systems show power-law spectra that extend at least up to $\sim 600 \mathrm{keV}$ (GRS 1915+105, GRO J1655-40; Grove et al. 1998) or even a few MeV (Cyg X-1; McConnell et al. 2000). This is higher than the 
cutoff energy in our spectra. These systems, however, are also the ones that show radio emission, often due to a jet. Thus it is possible that a jet-related component may be needed in addition to bulk Comptonization. Higher cutoff energies would also result for accretion onto a rapidly rotating hole, where the inner edge of the accretion disk is closer to the horizon. To explore this possibility a more realistic calculation of the GR effects is required than we have used here.

In conclusion, we have discussed for the first time the Comptonization by orbital motion in an accretion disk around a Schwarzschild black hole (as opposed to thermal Comptonization in a hot corona or bulk-motion Comptonization by radially-falling electrons) and have shown that high-energy power-law X-ray spectra of the type seen in the soft/high states of black hole accreters can be produced in this way.

Acknowledgements. The authors acknowledge partial support from the European Union Training and Mobility of Researchers Network Grant ERBFMRX/CT98/0195.

\section{References}

Cashwell, E. D., \& Everett, C. J. 1959, in A Practical Manual on the Monte Carlo Method for Random Walk Problems (Pergamon, Oxford)
Galeev, A. A., Rosner, R., \& Vaiana, G. S. 1979, ApJ, 229, 318

Grove, J. E., Johnson, W. N., Kroeger, R. A., et al. 1998, ApJ, 500,899

Hanawa, T. 1990, ApJ, 355, 585

Kylafis, N. D., \& Reig, P. 1999, in The neutron star - black hole connection, NATO ASI Ser.

Laurent, P., \& Titarchuk, L. 1999, ApJ, 511, 289

McConnell, M., Ryan, J. M., Collmar, W., et al. 2000, ApJ, 543,928

Misner, C. W., Thorne, K. S., \& Wheeler, J. A. 1973, in Gravitation (Freeman), 671

Miyamoto, S., Iga, S., Kitamoto, S., \& Kamado, Y. 1993, ApJ, 403, L39

Papathanassiou, H., \& Psaltis, D. 2001, MNRAS, in press [astro-ph/0011447]

Pozdnyakov, L. A., Sobol, I. M., \& Sunyaev, R. A. 1983, Astrophys. Space Phys. Rev., 2, 189

Poutanen, J. 1998, in Theory of black-hole accretion disks (Cambridge University Press)

Shapiro, S. L., \& Teukolsky, S. A. 1983, in Black holes, white dwarfs and neutron stars: The physics of compact objects (Wiley-Interscience), 341

Tanaka, Y., \& Lewin, W. H. G. 1995, in X-ray Binaries, ed. W. H. G. Lewin, J. van Paradijs, \& E. P. J. van den Heuvel (Cambridge University Press), 126

Tanaka, Y., \& Shibazaki, N. 1996, ARA\&A, 34, 607

Titarchuk, L., Mastichiadis, A., \& Kylafis, N. D. 1997, ApJ, 487,834 